\title{
Soliton Solutions of the Coupled Schrödinger-Boussinesq Equations for Kerr Law Nonlinearity
}

\author{
Anwar Ja'afar Mohamad Jawad (iD) and Mahmood Jawad Abu-AlShaeer \\ Al-Rafidain University College, Baghdad, Iraq \\ Correspondence should be addressed to Anwar Ja’afar Mohamad Jawad; anwar_jawad2001@yahoo.com
}

Received 8 September 2017; Accepted 26 October 2017; Published 1 January 2018

Academic Editor: Changbum Chun

Copyright (c) 2018 Anwar Jaafar Mohamad Jawad and Mahmood Jawad Abu-AlShaeer. This is an open access article distributed under the Creative Commons Attribution License, which permits unrestricted use, distribution, and reproduction in any medium, provided the original work is properly cited.

\begin{abstract}
In this paper, the coupled Schrödinger-Boussinesq equations (SBE) will be solved by the sech, tanh, csch, and the modified simplest equation method (MSEM). We obtain exact solutions of the nonlinear for bright, dark, and singular 1-soliton solution. Kerr law nonlinearity media are studied. Results have proven that modified simple equation method does not produce the soliton solution in general case. Solutions may find practical applications and will be important for the conservation laws for dispersive optical solitons.
\end{abstract}

\section{Introduction}

All optical communications are being used for transcontinental and transoceanic data transfer, through long-haul optical fibers, at the present time. There are various aspects of soliton communication that still need to be addressed. One of the features is the dispersive optical solitons. In presence of higher order dispersion terms, soliton communications are sometimes a hindrance as these dispersion terms produce soliton radiation. Nonlinear evolution equations have a major role in various scientific and engineering fields, such as optical fibers. Nonlinear wave phenomena of dispersion, dissipation, diffusion, reaction, and convection are very important in nonlinear wave equations. In recent years, quite a few methods for obtaining explicit traveling and solitary wave solutions of nonlinear evolution equations have been proposed. In recent years, exact homoclinic and heteroclinic solutions were proposed for some NEEs like nonlinear Schrödinger equation, Sine-Gordon equation, DaveyStewartson equation, Zakharov equation, and Boussinesq equation [1-7].

In particular, the study of the coupled SchrödingerBoussinesq equations has attracted much attention of mathematicians and physicists [8-10]. The existence of the global solution of the initial boundary problem for the equations was investigated in [8]. The existence of a periodic solution for the equations was considered in [9]. Kilicman and Abazari [10] used the $\left(G^{\prime} / G\right)$-expansion method to construct periodic and soliton solutions for the Schrödinger-Boussinesq. The investigation of nonlinear partial differential equations plays an important role in the study of nonlinear physical phenomena [9-12].

The nonlinear coupled Schrödinger-Boussinesq equation (SBE) governs the propagation of optical solitons in a dispersive optical fiber and is a very important equation in the area of theoretical and mathematical physics. This paper is going to take a look at the bright, dark, and singular soliton solutions for Kerr law nonlinearity media.

\section{Governing Equations}

Consider the coupled Schrödinger-Boussinesq equations (SBE). They appeared in [13] as a special case of general systems governing the stationary propagation of coupled nonlinear upper hybrid and magneto sonic waves in magnetized plasma. These equations were in the form [14]

$$
\begin{aligned}
& i \frac{\partial E}{\partial t}+\frac{\partial^{2} E}{\partial x^{2}}+\alpha_{1} E-N E=0, \\
& 3 \frac{\partial^{2} N}{\partial t^{2}}-\frac{\partial^{4} N}{\partial x^{4}}+3 \frac{\partial^{2}}{\partial x^{2}}\left(N^{2}\right)+\alpha_{2} \frac{\partial^{2} N}{\partial x^{2}}-\frac{\partial^{2}}{\partial x^{2}}\left(|E|^{2}\right) \\
& \quad=0
\end{aligned}
$$


where $\alpha_{1}, \alpha_{2}$ are real constants, $E(x, t)$ is a complex function, and $N(x, t)$ is a real function. The complete integrability of (1) was studied by Chowdhury et al. [15], and $N$-soliton solution, homoclinic orbit solution, and rogue solution were obtained by $\mathrm{Hu}$ et al. [16], Dai et al. [17-19], and Mu and Qin [20].

\section{The Traveling Solution}

Consider the nonlinear partial differential equation in the form

$$
F\left(u, u_{t}, u_{x}, u_{t t}, u_{x x}, \ldots\right)=0
$$

where $u(x, t)$ is a traveling wave solution of nonlinear partial differential equation (2). We use the transformations

$$
u(x, t)=f(\xi)
$$

where $\xi=x-\rho t+\chi$. This enables us to use the following changes:

$$
\begin{aligned}
& \frac{\partial}{\partial t}(\cdot)=-\rho \frac{d}{d \xi}(\cdot), \\
& \frac{\partial}{\partial x}(\cdot)=\frac{d}{d \xi}(\cdot) .
\end{aligned}
$$

Using (4) to transfer the nonlinear partial differential equation (2) to nonlinear ordinary differential equation,

$$
Q\left(f, f^{\prime}, f^{\prime \prime}, f^{\prime \prime \prime}, \ldots\right)=0 .
$$

The ordinary differential equation (5) is then integrated as long as all terms contain derivatives, where we neglect the integration constants.

\section{Hyperbolic Function Methods}

The solutions of many nonlinear equations can be expressed in the following form.

\subsection{Sech Function Method (Bright Soliton) [21]}

$$
\begin{aligned}
& f(\xi)=A \operatorname{sech}^{\beta}(\mu \xi), \\
& f^{\prime}(\xi)=-A \beta \mu \operatorname{sech}^{\beta}(\mu \xi) \cdot \tanh (\mu \xi), \\
& f^{\prime \prime}(\xi)=-A \beta \mu^{2}\left[(\beta+1) \operatorname{sech}^{\beta+2}(\mu \xi)\right. \\
& \left.-\beta \operatorname{sech}^{\beta}(\mu \xi)\right], \\
& f^{\prime \prime \prime}(\xi)=A \beta \mu^{3}\left[(\beta+1)(\beta+2) \operatorname{sech}^{\beta+2}(\mu \xi)\right. \\
& \left.-\beta^{2} \operatorname{sech}^{\beta}(\mu \xi)\right] \tanh (\mu \xi) .
\end{aligned}
$$

\subsection{Tanh Function Method (Dark Soliton) [22]}

$$
\begin{aligned}
& f(\xi)=A \tanh ^{\beta}(\mu \xi), \quad|\xi| \leq \frac{\pi}{2 \mu} \\
& f^{\prime}(\xi)=A \beta \mu\left[\tanh ^{\beta-1}(\mu \xi)-\tanh ^{\beta+1}(\mu \xi)\right],
\end{aligned}
$$

$$
\begin{aligned}
& f^{\prime \prime}(\xi)=A \beta \mu^{2}\left[(\beta-1) \tanh ^{\beta-2}(\mu \xi)-2 \beta \tanh ^{\beta}(\mu \xi)\right. \\
& \left.\quad+(\beta+1) \tanh ^{\beta+2}(\mu \xi)\right], \\
& f^{\prime \prime \prime}(\xi)=A \beta \mu^{3}\left[(\beta-1)(\beta-2) \tanh ^{\beta-3}(\mu \xi)\right. \\
& \quad-\{(\beta-1)(\beta-2)+2 \beta\} \tanh ^{\beta-1}(\mu \xi) \\
& \quad+\{(\beta+1)(\beta+2)+2 \beta\} \tanh ^{\beta+1}(\mu \xi) \\
& \left.\quad-(\beta+1)(\beta+2) \tanh ^{\beta+3}(\mu \xi)\right] .
\end{aligned}
$$

\subsection{Csch Function Method (Singular Soliton) [21, 22]}

$$
\begin{aligned}
& f(\xi)=A \operatorname{csch}^{\beta}(\mu \xi), \\
& f^{\prime}(\xi)=-A \beta \mu \operatorname{csch}^{\beta}(\mu \xi) \cdot \operatorname{coth}(\mu \xi), \\
& f^{\prime \prime}(\xi)=A \beta \mu^{2}\left[(\beta+1) \operatorname{csch}^{\beta+2}(\mu \xi)+\beta \operatorname{csch}^{\beta}(\mu \xi)\right], \\
& f^{\prime \prime \prime}(\xi)=-A \beta \mu^{3}\left[(\beta+1)(\beta+2) \operatorname{csch}^{\beta+2}(\mu \xi)\right. \\
& \left.\quad+\beta^{2} \operatorname{csch}^{\beta}(\mu \xi)\right] \operatorname{coth}(\mu \xi),
\end{aligned}
$$

where $A$ represent the amplitudes of the solitons and $\mu$ represents the solitons width.

We substitute (6), (7), or (8) into the reduced equation (5), balance the terms of the sech, tanh, and csch functions, and solve the resulting system of algebraic equations by using computerized symbolic packages. We next collect all terms with the same power in $\operatorname{sech}^{k}(\mu \xi), \tanh ^{k}(\mu \xi), \operatorname{orcsch}^{k}(\mu \xi)$, set to zero their coefficients to get a system of algebraic equations among the unknowns $A, \mu$, and $\beta$, and solve the subsequent system.

\section{The Application}

The starting hypothesis for solving (1) by the aid of traveling waves solution is as follows: introduce the transformations

$$
\begin{aligned}
& E(x, t)=e^{i \theta(x, t)} u(\xi), \\
& N(x, t)=v(\xi),
\end{aligned}
$$

where

$$
\begin{aligned}
& \theta=k x+\omega t+\epsilon_{0}, \\
& \xi=(x-\rho t+\chi),
\end{aligned}
$$

where $k, \omega, \rho, \epsilon_{0}$, and $\chi$ are real constants. The parameter $\rho$ represents the soliton velocity.

Substituting (9) and (10) into (1) and decomposing into real and imaginary parts leads to

$$
\begin{array}{r}
u^{\prime \prime}-\left[\omega+k^{2}-\alpha_{1}\right] u-u v=0, \\
2 k-\rho=0, \\
{\left[12 k^{2}+\alpha_{2}\right] v^{\prime \prime}-v^{\prime \prime \prime \prime}+3\left(v^{2}\right)^{\prime \prime}-\left(u^{2}\right)^{\prime \prime}=0 .}
\end{array}
$$


Substitute (12) in (10), then

$$
\xi=(x-2 k t+\chi)
$$

Integrating (13) twice with zero constant, (13) can be written as

$$
\left[12 k^{2}+\alpha_{2}\right] v-v^{\prime \prime}+3 v^{2}-u^{2}=0
$$

5.1. Bright Soliton. Seeking the solution by sech function method as in (6)

$$
\begin{aligned}
& u(\xi)=A_{1} \operatorname{sech}^{\beta_{1}}(\mu \xi), \\
& v(\xi)=A_{2} \operatorname{sech}^{\beta_{2}}(\mu \xi),
\end{aligned}
$$

the system of equations in (11) and (15) becomes, respectively,

$$
\begin{aligned}
& \beta_{1} \mu^{2}\left[\left(\beta_{1}+1\right) \operatorname{sech}^{\beta_{1}+2}(\mu \xi)-\beta_{1} \operatorname{sech}^{\beta_{1}}(\mu \xi)\right]+[\omega \\
& \left.+k^{2}-\alpha_{1}\right] \operatorname{sech}^{\beta_{1}}(\mu \xi)-A_{2} \operatorname{sech}^{\beta_{1}+\beta_{2}}(\mu \xi)=0, \\
& {\left[12 k^{2}+\alpha_{2}\right] A_{2} \operatorname{sech}^{\beta_{2}}(\mu \xi)} \\
& +A_{2} \beta_{2} \mu^{2}\left[\left(\beta_{2}+1\right) \operatorname{sech}^{\beta_{2}+2}(\mu \xi)\right. \\
& \left.-\beta_{2} \operatorname{sech}^{\beta_{2}}(\mu \xi)\right]+3 A_{2}{ }^{2} \operatorname{sech}^{2 \beta_{2}}(\mu \xi) \\
& -A_{1}{ }^{2} \operatorname{sech}^{2 \beta_{1}}(\mu \xi)=0 .
\end{aligned}
$$

Equating the exponents and the coefficients of each pair of the sech functions, we find

$$
\begin{aligned}
2 \beta_{1} & =\beta_{2}+2, \\
\beta_{1}+\beta_{2} & =\beta_{1}+2, \text { then } \beta_{1}=\beta_{2}=2 .
\end{aligned}
$$

Thus setting coefficients of (17)-(18) to zero yields set system of equations:

$$
\begin{aligned}
4 \mu^{2}-\left[\omega+k^{2}-\alpha_{1}\right] & =0 \\
{\left[12 k^{2}+\alpha_{2}\right]-4 \mu^{2} } & =0 \\
6 \mu^{2}-A_{2} & =0 \\
6 A_{2} \mu^{2}+3 A_{2}^{2}-A_{1}^{2} & =0
\end{aligned}
$$

Solving the system of equations in (20), we get

$$
\begin{aligned}
& A_{1}=\mp 3\left[12 k^{2}+\alpha_{2}\right], \\
& A_{2}=\frac{3}{2}\left[12 k^{2}+\alpha_{2}\right],
\end{aligned}
$$

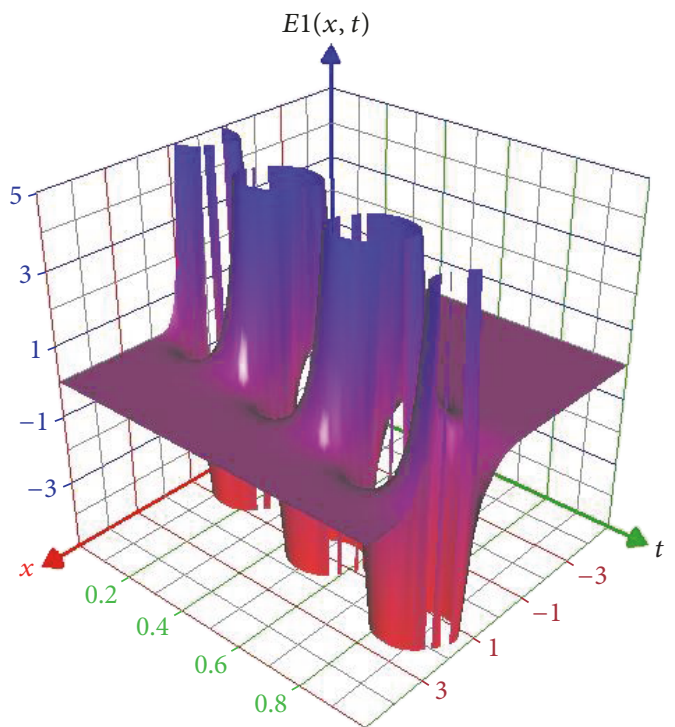

Figure 1: The solitary wave of the real part of $E_{1}(x, t)$ in (23) for $-5 \leq x \leq 5,0 \leq t \leq 1$.

$$
\begin{aligned}
& \mu=\mp \sqrt{\frac{\left[12 k^{2}+\alpha_{2}\right]}{4}}, \\
& \omega=\left[11 k^{2}+\alpha_{1}+\alpha_{2}\right] \text {, } \\
& E_{1}(x, t)=\mp 3 e^{i\left(k x+\left(11 k^{2}+\alpha_{1}+\alpha_{2}\right) t+\epsilon_{0}\right)}\left[12 k^{2}+\alpha_{2}\right] \\
& \cdot \operatorname{sech}^{2}\left(\sqrt{\frac{\left[12 k^{2}+\alpha_{2}\right]}{4}}(x-2 k t+\chi)\right), \\
& N_{1}(x, t)=\frac{3}{2}\left[12 k^{2}+\alpha_{2}\right] \\
& \cdot \operatorname{sech}^{2}\left(\sqrt{\frac{\left[12 k^{2}+\alpha_{2}\right]}{4}}(x-2 k t+\chi)\right) \text {. }
\end{aligned}
$$

For $k=\alpha_{1}=1, \alpha_{2}=4, \epsilon_{0}=\chi=0$, the real part of $E_{1}(x, t)=$ $48 \cos (x+16 t) \operatorname{sech}^{2}\{2(x-2 t)\}$, and $N_{1}(x, t)=24 \operatorname{sech}^{2}\{2(x-$ $2 t)\}$.

Figures 1 and 2 represent the solitary wave of the real part of $E_{1}(x, t)$ in (23) and $N_{1}(x, t)$ in (24), respectively, for $-5 \leq$ $x \leq 5,0 \leq t \leq 1$.

5.2. Dark Soliton. Seeking the solution by tanh function method as in (7)

$$
\begin{aligned}
& u(\xi)=A_{1} \tanh ^{\beta_{1}}(\mu \xi), \\
& v(\xi)=A_{2} \tanh ^{\beta_{2}}(\mu \xi),
\end{aligned}
$$




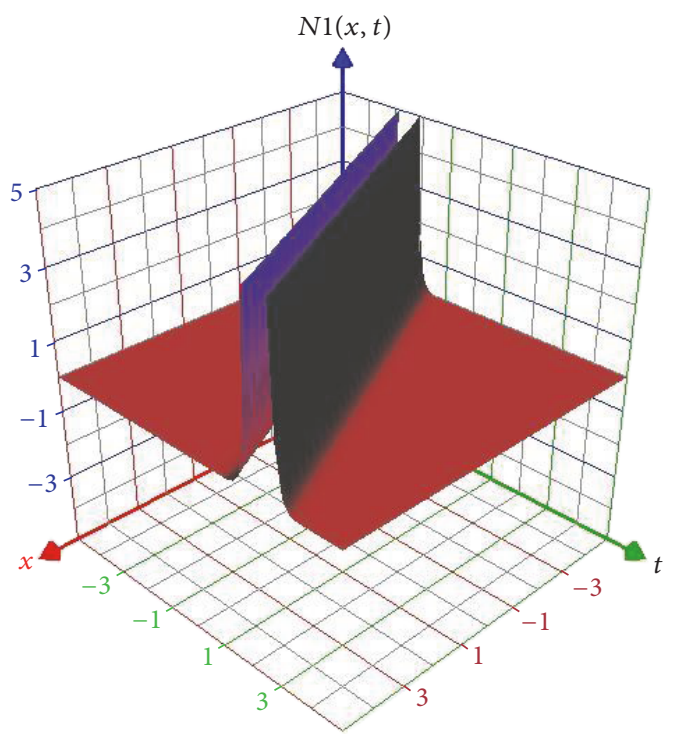

FIgURE 2: The solitary wave $N_{1}(x, t)$ in (24) for $-5 \leq x \leq 5,0 \leq t \leq$ 1.

the system of equations in (11) and (15) becomes, respectively,

$$
\begin{gathered}
\beta_{1} \mu^{2}\left[\left(\beta_{1}-1\right) \tanh ^{\beta_{1}-2}(\mu \xi)-2 \beta_{1} \tanh ^{\beta_{1}}(\mu \xi)\right. \\
\left.+\left(\beta_{1}+1\right) \tanh ^{\beta_{1}+2}(\mu \xi)\right]-\left[\omega+k^{2}-\alpha_{1}\right] \\
\cdot \tanh ^{\beta_{1}}(\mu \xi)-A_{2} \tanh ^{\beta_{1}+\beta_{2}}(\mu \xi)=0, \\
{\left[12 k^{2}+\alpha_{2}\right] A_{2} \tanh ^{\beta_{2}}(\mu \xi)} \\
-A_{2} \beta_{2} \mu^{2}\left[\left(\beta_{2}-1\right) \tanh ^{\beta_{2}-2}(\mu \xi)\right. \\
\left.-2 \beta_{2} \tanh ^{\beta_{2}}(\mu \xi)+\left(\beta_{2}+1\right) \tanh ^{\beta_{2}+2}(\mu \xi)\right] \\
+3 A_{2}{ }^{2} \tanh ^{2 \beta_{2}}(\mu \xi)-A_{1}{ }^{2} \tanh ^{2 \beta_{1}}(\mu \xi)=0 .
\end{gathered}
$$

Equating the exponents and the coefficients of each pair of the sech functions, we find

$$
\begin{aligned}
2 \beta_{1} & =\beta_{2}+2, \\
\beta_{1}+\beta_{2} & =\beta_{1}+2, \text { then } \beta_{1}=\beta_{2}=2 .
\end{aligned}
$$

Thus setting coefficients of (26)-(27) to zero yields set system of equations:

$$
\begin{aligned}
8 \mu^{2}+\left[\omega+k^{2}-\alpha_{1}\right] & =0, \\
{\left[12 k^{2}+\alpha_{2}\right]+8 \mu^{2} } & =0, \\
6 \mu^{2}-A_{2} & =0, \\
-6 A_{2} \mu^{2}+3 A_{2}{ }^{2}-A_{1}{ }^{2} & =0 .
\end{aligned}
$$

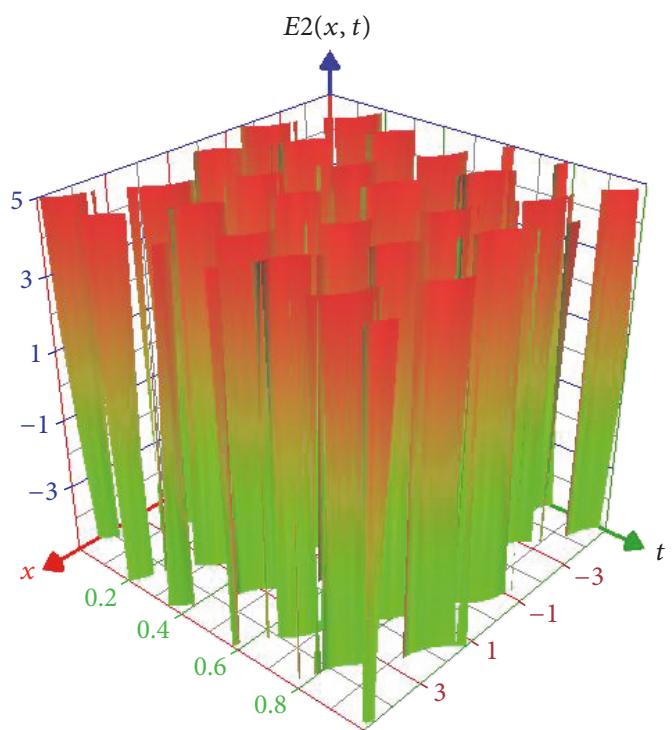

FIGURE 3: The solitary wave of the real part of $E_{2}(x, t)$ in (32) for $-5 \leq x \leq 5,0 \leq t \leq 1$.

Solving the system of equations in (29), we get

$$
\begin{aligned}
& \omega=\left[11 k^{2}+\alpha_{1}+\alpha_{2}\right], \\
& \begin{array}{l}
\mu=\mp i \sqrt{\frac{\left[12 k^{2}+\alpha_{2}\right]}{8}}, \\
A_{1}= \pm \frac{3 \sqrt{2}}{4}\left[12 k^{2}+\alpha_{2}\right], \\
A_{2}=\frac{-3}{4}\left[12 k^{2}+\alpha_{2}\right], \\
E_{2}(x, t)= \pm e^{i\left(k x+\left(11 k^{2}+\alpha_{1}+\alpha_{2}\right) t+\epsilon_{0}\right)} 3 \sqrt{2} \frac{\left[12 k^{2}+\alpha_{2}\right]}{4} \\
\quad \cdot \sec ^{2}\left(\sqrt{\frac{\left[12 k^{2}+\alpha_{2}\right]}{8}}(x-2 k t+\chi)\right), \\
N_{2}(x, t)=-3 \frac{\left[12 k^{2}+\alpha_{2}\right]}{4} \\
\quad \cdot \sec ^{2}\left(\sqrt{\frac{\left[12 k^{2}+\alpha_{2}\right]}{8}}(x-2 k t+\chi)\right) .
\end{array}
\end{aligned}
$$

For $k=\alpha_{1}=1, \alpha_{2}=4, \epsilon_{0}=\chi=0$, the real part of $E_{2}(x$, $t)=12 \sqrt{2} \cos (x+16 t) \sec ^{2}\{\sqrt{2}(x-2 t)\}$, and $N_{2}(x, t)=$ $-12 \sec ^{2}\{\sqrt{2}(x-2 t)\}$.

Figures 3 and 4 represent the solitary wave of the real part of $E_{2}(x, t)$ in (32) and $N_{2}(x, t)$ in (33) for $-5 \leq x \leq 5,0 \leq t \leq$ 1. 


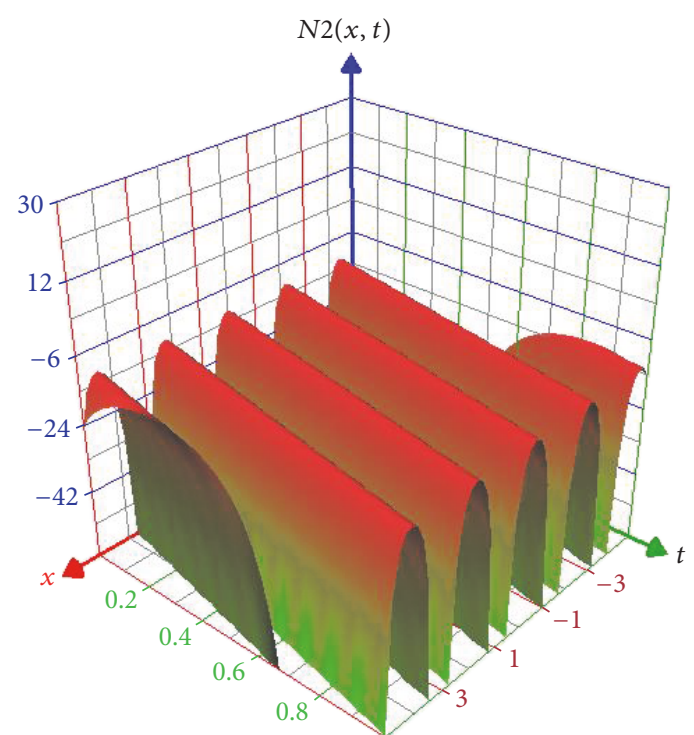

Figure 4: The solitary wave $N_{2}(x, t)$ in (33) for $-5 \leq x \leq 5,0 \leq t \leq$ 1 .

5.3. Singular Soliton. Seeking the solution by sech function method as in (8)

$$
\begin{aligned}
& u(\xi)=A_{1} \operatorname{coth}^{\beta_{1}}(\mu \xi), \\
& v(\xi)=A_{2} \operatorname{coth}^{\beta_{2}}(\mu \xi),
\end{aligned}
$$

the system of equations in (11) and (15) becomes, respectively,

$$
\begin{aligned}
& \beta_{1} \mu^{2}\left[\left(\beta_{1}+1\right) \operatorname{coth}^{\beta_{1}+2}(\mu \xi)+\beta_{1} \operatorname{coth}^{\beta_{1}}(\mu \xi)\right]+[\omega \\
& \left.+k^{2}-\alpha_{1}\right] \operatorname{coth}^{\beta_{1}}(\mu \xi)-A_{2} \operatorname{coth}^{\beta_{1}+\beta_{2}}(\mu \xi)=0, \\
& {\left[12 k^{2}+\alpha_{2}\right] A_{2} \operatorname{coth}^{\beta_{2}}(\mu \xi)} \\
& +A_{2} \beta_{2} \mu^{2}\left[\left(\beta_{2}+1\right) \operatorname{coth}^{\beta_{2}+2}(\mu \xi)\right. \\
& \left.+\beta_{2} \operatorname{coth}^{\beta_{2}}(\mu \xi)\right]+3 A_{2}{ }^{2} \operatorname{coth}^{2 \beta_{2}}(\mu \xi) \\
& \quad-A_{1}{ }^{2} \operatorname{coth}^{2 \beta_{1}}(\mu \xi)=0 .
\end{aligned}
$$

Equating the exponents and the coefficients of each pair of the sech functions, we find

$$
\begin{aligned}
2 \beta_{1} & =\beta_{2}+2, \\
\beta_{1}+\beta_{2} & =\beta_{1}+2, \text { then } \beta_{1}=\beta_{2}=2 .
\end{aligned}
$$

Thus setting coefficients of (35)-(36) to zero yields set system of equations:

$$
\begin{aligned}
4 \mu^{2}+\left[\omega+k^{2}-\alpha_{1}\right] & =0 \\
{\left[12 k^{2}+\alpha_{2}\right]+4 \mu^{2} } & =0 \\
6 \mu^{2}-A_{2} & =0 \\
6 A_{2} \mu^{2}+3 A_{2}{ }^{2}-A_{1}{ }^{2} & =0
\end{aligned}
$$

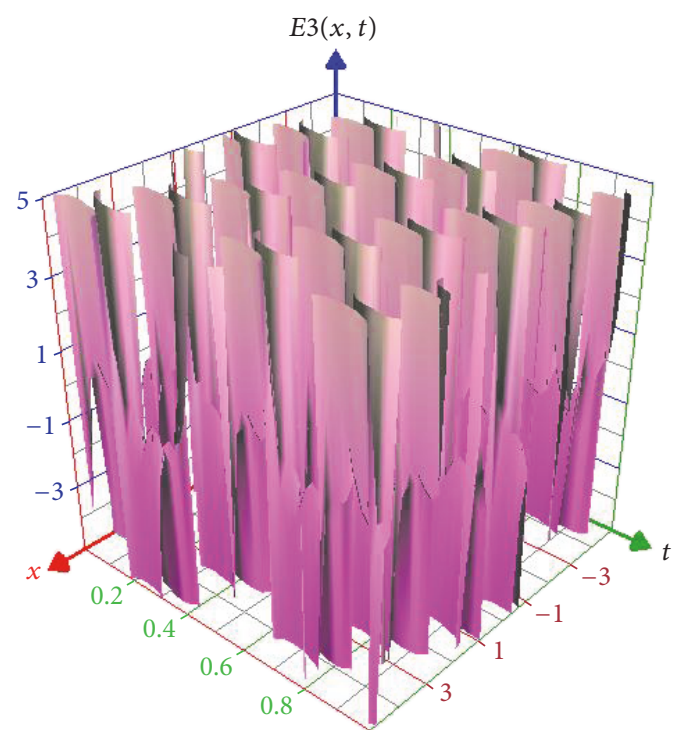

FIGURE 5: The solitary wave of the real part of $E_{3}(x, t)$ in (40) for $-5 \leq x \leq 5,0 \leq t \leq 1$.

Solving the system of equations in (38), we get

$$
\begin{aligned}
& A_{1}= \pm 3\left[12 k^{2}+\alpha_{2}\right] \text {, } \\
& A_{2}=-3 \frac{\left[12 k^{2}+\alpha_{2}\right]}{2} \text {, } \\
& \mu=\mp i \sqrt{\frac{\left[12 k^{2}+\alpha_{2}\right]}{4}}, \\
& \omega=11 k^{2}+\alpha_{1}+\alpha_{2}, \\
& E_{3}(x, t)=\mp e^{i\left(k x+\left(11 k^{2}+\alpha_{1}+\alpha_{2}\right) t+\epsilon_{0}\right)} 6\left(6 k^{2}+1\right) \\
& \cdot \cot ^{2}\left(\sqrt{\frac{6 k^{2}+1}{2}}(x-2 k t+\chi)\right) \text {, } \\
& N_{3}(x, t)=3\left(6 k^{2}+1\right) \\
& \cdot \cot ^{2}\left(\sqrt{\frac{6 k^{2}+1}{2}}(x-2 k t+\chi)\right) \text {. }
\end{aligned}
$$

For $k=\alpha_{1}=1, \alpha_{2}=4, \epsilon_{0}=\chi=0$, the real part of $E_{3}(x, t)=42 \cos (x+16 t) \cot ^{2}\{\sqrt{7 / 2}(x-2 t)\}$, and $N_{3}(x, t)=$ $21 \cot ^{2}\{\sqrt{7 / 2}(x-2 t)\}$.

Figures 5 and 6 represent the solitary wave of the real part of $E_{3}(x, t)$ in (40) and $N_{3}(x, t)$ in (41) for $-5 \leq x \leq 5,0 \leq t \leq$ 1.

5.4. Modified Simple Equation Method. This section will analyze (11) and (15) by the modified simple equation method; assume that solutions are of the form [23] 


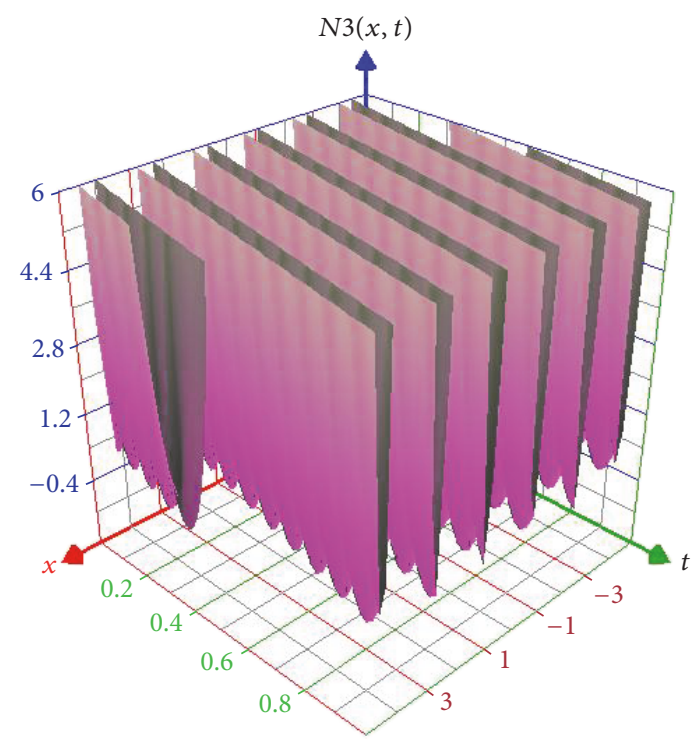

FIGURE 6: The solitary wave of $N_{3}(x, t)$ in (41) for $-5 \leq x \leq 5,0 \leq$ $t \leq 1$.

$$
\begin{aligned}
& u(\xi)=\sum_{i=0}^{m} A_{i}\left(\frac{\psi_{\xi}}{\psi}\right)^{i}, \\
& v(\xi)=\sum_{i=0}^{n} B_{i}\left(\frac{\psi_{\xi}}{\psi}\right)^{i},
\end{aligned}
$$

where the parameters $m, n$ can be found by balancing the highest-order linear term with the nonlinear terms in (11) and (15), respectively.

In (11), we balance $u^{\prime \prime}$ with $u v$, to obtain $m+2=m+n$, and then $n=2$. While in (15), We balance $v^{\prime \prime}$ with $u^{2}$, to obtain $n+2=2 m$, and then $m=2$.

Then

$$
\begin{aligned}
u(\xi)= & A_{0}+A_{1} \frac{\psi_{\xi}}{\psi}+A_{2} \frac{\psi_{\xi}^{2}}{\psi^{2}} \\
u_{\xi}= & A_{1} \frac{\psi_{\xi \xi}}{\psi}-A_{1} \frac{\psi_{\xi}^{2}}{\psi^{2}}+2 A_{2} \frac{\psi_{\xi} \psi_{\xi \xi}}{\psi^{2}}-2 A_{2} \frac{\psi_{\xi}^{3}}{\psi^{3}} \\
u_{\xi \xi}= & A_{1} \frac{\psi_{\xi \xi \xi}}{\psi}+2 A_{2} \frac{\psi_{\xi \xi}^{2}}{\psi^{2}}+2 A_{2} \frac{\psi_{\xi \xi \xi} \psi_{\xi}}{\psi^{2}} \\
& -3 A_{1} \frac{\psi_{\xi} \psi_{\xi \xi}}{\psi^{2}}-10 A_{2} \frac{\psi_{\xi \xi} \psi_{\xi}^{2}}{\psi^{3}}-2 A_{1} \frac{\psi_{\xi}^{3}}{\psi^{3}} \\
& -2 A_{2} \frac{\psi_{\xi}}{\psi^{4}}, \\
v(\xi)= & B_{0}+B_{1} \frac{\psi_{\xi}}{\psi}+B_{2} \frac{\psi_{\xi}^{2}}{\psi^{2}}, \\
v_{\xi}= & B_{1} \frac{\psi_{\xi \xi}}{\psi}-B_{1} \frac{\psi_{\xi}^{2}}{\psi^{2}}+2 B_{2} \frac{\psi_{\xi} \psi_{\xi \xi}}{\psi^{2}}-2 B_{2} \frac{\psi_{\xi}^{3}}{\psi^{3}}
\end{aligned}
$$

$$
\begin{aligned}
v_{\xi \xi}= & B_{1} \frac{\psi_{\xi \xi \xi}}{\psi}+2 B_{2} \frac{\psi_{\xi \xi}^{2}}{\psi^{2}}+2 B_{2} \frac{\psi_{\xi \xi \xi} \psi_{\xi}}{\psi^{2}}-3 B_{1} \frac{\psi_{\xi} \psi_{\xi \xi}}{\psi^{2}} \\
& -10 B_{2} \frac{\psi_{\xi \xi} \psi_{\xi}^{2}}{\psi^{3}}-2 B_{1} \frac{\psi_{\xi}^{3}}{\psi^{3}}-2 B_{2} \frac{\psi_{\xi}^{4}}{\psi^{4}}
\end{aligned}
$$

where $A_{0}, A_{1}, A_{2}, B_{0}, B_{1}$, and $B_{2}$ are constants to be calculated.

Substitute (43) in (11) and (15), respectively, to get

$$
\begin{aligned}
& {\left[A_{1} \frac{\psi_{\xi \xi \xi}}{\psi}+2 A_{2} \frac{\psi_{\xi \xi}^{2}}{\psi^{2}}+2 A_{2} \frac{\psi_{\xi \xi \xi} \psi_{\xi}}{\psi^{2}}-3 A_{1} \frac{\psi_{\xi} \psi_{\xi \xi}}{\psi^{2}}\right.} \\
& \left.-10 A_{2} \frac{\psi_{\xi \xi} \psi_{\xi}^{2}}{\psi^{3}}-2 A_{1} \frac{\psi_{\xi}^{3}}{\psi^{3}}-2 A_{2} \frac{\psi_{\xi}^{4}}{\psi^{4}}\right]-\left[\omega+k^{2}\right.
\end{aligned}
$$$$
\left.-\alpha_{1}\right]\left[A_{0}+A_{1} \frac{\psi_{\xi}}{\psi}+A_{2} \frac{\psi_{\xi}^{2}}{\psi^{2}}\right]-B_{0}\left(A_{0}+A_{1} \frac{\psi_{\xi}}{\psi}\right.
$$$$
\left.+A_{2} \frac{\psi_{\xi}^{2}}{\psi^{2}}\right)-B_{1}\left(A_{0} \frac{\psi_{\xi}}{\psi}+A_{1} \frac{\psi_{\xi}^{2}}{\psi^{2}}+A_{2} \frac{\psi_{\xi}^{3}}{\psi^{3}}\right)
$$$$
-B_{2}\left(A_{0} \frac{\psi_{\xi}^{2}}{\psi^{2}}+A_{1} \frac{\psi_{\xi}^{3}}{\psi^{3}}+A_{2} \frac{\psi_{\xi}^{4}}{\psi^{4}}\right)=0,
$$

$$
\left[12 k^{2}+\alpha_{2}\right]\left[B_{0}+B_{1} \frac{\psi_{\xi}}{\psi}+B_{2} \frac{\psi_{\xi}^{2}}{\psi^{2}}\right]-\left[B_{1} \frac{\psi_{\xi \xi \xi}}{\psi}\right.
$$$$
+2 B_{2} \frac{\psi_{\xi \xi}^{2}}{\psi^{2}}+2 B_{2} \frac{\psi_{\xi \xi \xi} \psi_{\xi}}{\psi^{2}}-3 B_{1} \frac{\psi_{\xi} \psi_{\xi \xi}}{\psi^{2}}
$$

$$
\left.-10 B_{2} \frac{\psi_{\xi \xi} \psi_{\xi}^{2}}{\psi^{3}}-2 B_{1} \frac{\psi_{\xi}^{3}}{\psi^{3}}-2 B_{2} \frac{\psi_{\xi}^{4}}{\psi^{4}}\right]+3 B_{0}\left(B_{0}\right.
$$$$
\left.+B_{1} \frac{\psi_{\xi}}{\psi}+B_{2} \frac{\psi_{\xi}^{2}}{\psi^{2}}\right)+3 B_{1}\left(B_{0} \frac{\psi_{\xi}}{\psi}+B_{1} \frac{\psi_{\xi}^{2}}{\psi^{2}}\right.
$$$$
\left.+B_{2} \frac{\psi_{\xi}^{3}}{\psi^{3}}\right)+3 B_{2}\left(B_{0} \frac{\psi_{\xi}^{2}}{\psi^{2}}+B_{1} \frac{\psi_{\xi}^{3}}{\psi^{3}}+B_{2} \frac{\psi_{\xi}^{4}}{\psi^{4}}\right)
$$$$
-A_{0}\left(A_{0}+A_{1} \frac{\psi \xi}{\psi}+A_{2} \frac{\psi_{\xi}^{2}}{\psi^{2}}\right)-A_{1}\left(A_{0} \frac{\psi_{\xi}}{\psi}\right.
$$$$
\left.+A_{1} \frac{\psi_{\xi}^{2}}{\psi^{2}}+A_{2} \frac{\psi_{\xi}^{3}}{\psi^{3}}\right)-A_{2}\left(A_{0} \frac{\psi_{\xi}^{2}}{\psi^{2}}+A_{1} \frac{\psi_{\xi}^{3}}{\psi^{3}}\right.
$$$$
\left.+A_{2} \frac{\psi_{\xi}^{4}}{\psi^{4}}\right)=0
$$ 
In (44) equating expressions at $\left(\psi^{j} j=0,-1,-2,-3,-4\right)$ to zero, we get the following system of equations:

$$
\begin{aligned}
& \psi_{\xi \xi}+\frac{\left[2 A_{1}+A_{2} B_{1}+A_{1} B_{2}\right]}{10 A_{2}} \psi_{\xi}=0, \\
& 2 A_{2} \psi_{\xi \xi}^{2}+2 A_{2} \psi_{\xi \xi \xi} \psi_{\xi}-3 A_{1} \psi_{\xi} \psi_{\xi \xi} \\
& -\left\{\left[\omega+k^{2}-\alpha_{1}\right] A_{2}+A_{2} B_{0}+A_{1} B_{1}+A_{0} B_{2}\right\} \psi_{\xi}^{2} \\
& =0 \text {, } \\
& \psi_{\xi \xi \xi}-\frac{\left[\omega+k^{2}-\alpha_{1}\right] A_{1}-B_{0} A_{1}-B_{1} A_{0}}{A_{1}} \psi_{\xi}=0, \\
& {\left[\omega+k^{2}-\alpha_{1}\right]+B_{0}=0,} \\
& {\left[12 k^{2}+\alpha_{2}\right] B_{0}+3 B_{0}{ }^{2}-A_{0}{ }^{2}=0 \text {, }} \\
& 2 B_{2}+3 B_{2}^{2}-A_{2}^{2}=0 \text {, } \\
& B_{2}=-2 \text {, } \\
& \psi_{\xi \xi}+\frac{\left\{2 B_{1}+6 B_{1} B_{2}-2 A_{1} A_{2}\right\}}{10 B_{2}} \psi_{\xi}=0, \\
& 2 B_{2} \psi_{\xi \xi}^{2}+2 B_{2} \psi_{\xi \xi \xi} \psi_{\xi}-3 B_{1} \psi_{\xi} \psi_{\xi \xi}-\left\{\left[12 k^{2}+\alpha_{2}\right] B_{2}\right. \\
& \left.+6 B_{0} B_{2}+3 B_{1}^{2}-2 A_{0} A_{2}-A_{1}^{2}\right\} \psi_{\xi}^{2}=0, \\
& B_{1} \psi_{\xi \xi \xi}-\left\{\left[12 k^{2}+\alpha_{2}\right] B_{1}+6 B_{1} B_{0}-2 A_{0} A_{1}\right\} \psi_{\xi}=0 .
\end{aligned}
$$

Obviously when solving the system of (45), we conclude that equations can be satisfied simultaneously for the following constraints. Hence, the modified simple equation method does not produce the soliton solution in general case:

$$
\begin{aligned}
& A_{0}=0, \\
& B_{0}=0, \\
& B_{1}=A_{1}=0, \\
& A_{2}=2 \sqrt{2}, \\
& B_{2}=-2, \\
& \omega=\alpha_{1}-k^{2}, \\
& \alpha_{2}=-12 k^{2} .
\end{aligned}
$$

Then we will solve the following ordinary differential equation:

$$
\psi_{\xi \xi}=0
$$

and therefore

$$
\psi=a_{0}+a_{1} \xi
$$

where $a_{0}, a_{1}$ are arbitrary constants.
And

$$
\begin{aligned}
& u(x, t)=2 \sqrt{2} \frac{a_{1}^{2}}{\left[a_{0}+a_{1}(x-2 k t+\chi)\right]^{2}}, \\
& v(x, t)=-2 \frac{a_{1}^{2}}{\left[a_{0}+a_{1}(x-2 k t+\chi)\right]^{2}} .
\end{aligned}
$$

Finally solutions become

$$
\begin{aligned}
& E_{4}(x, t) \\
& \quad=2 \sqrt{2} e^{i\left(k x+\left\{\alpha_{1}-k^{2}\right\} t+\epsilon_{0}\right)}\left\{\frac{a_{1}^{2}}{\left[a_{0}+a_{1}(x-2 k t+\chi)\right]^{2}}\right\}, \\
& N_{4}(x, t)=-2\left\{\frac{a_{1}^{2}}{\left[a_{0}+a_{1}(x-2 k t+\chi)\right]^{2}}\right\} .
\end{aligned}
$$

\section{Conclusion}

In this paper the dispersive bright, dark, and singular soliton solutions to SBE with Kerr law of nonlinearity were studied. The sech, tanh, csch, and the modified simplest equation method have been successfully applied to find solitons solutions for the coupled Schrödinger-Boussinesq equations. Several constraint conditions were assuring the existence of such solitons with Kerr law nonlinearity. The modified simple equation method does not produce the soliton solution in general case. Solutions by three methods are plotted in figures for the real and imaginary parts for $E(x, t)$ and $N(x, t)$. Compatibility in figures shape between the solutions of $E(x, t)$ and $N(x, t)$ by the same method sometimes appeared. Solutions may be important for the conservation laws for dispersive optical solitons. Those research outcomes will be soon disseminated.

\section{Conflicts of Interest}

The authors declare that there are no conflicts of interest regarding the publication of this paper.

\section{Acknowledgments}

This work was supported by Al-Rafidain University College.

\section{References}

[1] M. J. Ablowitz and B. M. Herbst, "On homoclinic structure and numerically induced chaos for the nonlinear Schrödinger equation," SIAM Journal on Applied Mathematics, vol. 50, no. 2, pp. 339-351, 1990.

[2] N. Ercolani, M. G. Forest, and D. W. McLaughlin, "Geometry of the modulational instability. III. Homoclinic orbits for the periodic sine-Gordon equation," Physica D: Nonlinear Phenomena, vol. 43 , no. $2-3$, pp. $349-384,1990$

[3] Z. Dai and J. Huang, "Homoclinic tubes for the DaveyStewartson II equation with periodic boundary conditions," Chinese Journal of Physics, vol. 43, no. 2, pp. 349-356, 2005. 
[4] Z. Dai, J. Huang, M. Jiang, and S. Wang, "Homoclinic orbits and periodic solitons for Boussinesq equation with even constraint," Chaos, Solitons \& Fractals, vol. 26, no. 4, pp. 1189-1194, 2005.

[5] Z. Dai, J. Huang, and M. Jiang, "Explicit homoclinic tube solutions and chaos for Zakharov system with periodic boundary," Physics Letters A, vol. 352, no. 4-5, pp. 411-415, 2006.

[6] A. J. M. Jawad, "Soliton Solutions for Nonlinear Systems (2+1)Dimensional Equations," IOSR Journal of Mathematics, vol. 1, no. 6, pp. 27-34, 2012.

[7] Y. Hase and J. Satsuma, "An N-soliton solution for the nonlinear Schrödinger equation coupled to the Boussinesq equation," Journal of the Physical Society of Japan, vol. 57, no. 3, pp. 679682,1988 .

[8] B. L. Guo and L. J. Shen, "The global solution of initial value problem for nonlinear Schrödinger-Boussinesq equation in 3-dimensions," Acta Mathematicae Applicatae Sinica. English Series, vol. 6, no. 1, pp. 11-21, 1990.

[9] B. Guo and X. Du, "Existence of the periodic solution for the weakly damped Schrödinger-Boussinesq equation," Journal of Mathematical Analysis and Applications, vol. 262, no. 2, pp. 453472, 2001.

[10] A. Kilicman and R. Abazari, "Travelling wave solutions of the SchröDinger-Boussinesq system," Abstract and Applied Analysis, vol. 2012, Article ID 198398, 11 pages, 2012.

[11] S. Y. Lai and B. Wiwatanapataphe, "The asymptotics of global solutions for semilinear wave equations in two space dimensions," Dynamics of Continuous, Discrete \& Impulsive Systems. Series B. Applications \& Algorithms, vol. 18, no. 5, pp. 647-657, 2011.

[12] S. Lai, Y. H. Wu, and B. Wiwatanapataphee, "On exact travelling wave solutions for two types of nonlinear $k(n, n)$ equations and a generalized KP equation," Journal of Computational and Applied Mathematics, vol. 212, no. 2, pp. 291-299, 2008.

[13] R. Conte and M. Musette, "Link between solitary waves and projective Riccati equations," Journal of Physics A: Mathematical and General, vol. 25, no. 21, pp. 5609-5623, 1992.

[14] X. Huang, "The investigation of solutions to the coupled Schrödinger-Boussinesq equations," Abstract and Applied Analysis, vol. 2013, Article ID 170372, 5 pages, 2013.

[15] A. R. Chowdhury, B. Dasgupta, and N. N. Rao, "Painléve analysis and Backlund transformations for coupled generalized Schrödinger-Boussinesq system," Chaos, Solitons \& Fractals, vol. 9, no. 10, pp. 1747-1753, 1998.

[16] X.-B. Hu, B.-L. Guo, and H.-W. Tam, "Homoclinic orbits for the coupled Schrödinger-Boussinesq equation and coupled higgs equation," Journal of the Physical Society of Japan, vol. 72, no. 1, pp. 189-190, 2003.

[17] Z.-D. Dai, Z.-J. Liu, and D.-L. Li, "Exact periodic solitary-wave solution for KdV equation," Chinese Physics Letters, vol. 25, no. 5, pp. 1531-1533, 2008.

[18] Z. Dai, J. Liu, and D. Li, "Applications of HTA and EHTA to YTSF equation," Applied Mathematics and Computation, vol. 207, no. 2, pp. 360-364, 2009.

[19] Z. Dai, Z. Li, Z. Liu, and D. Li, "Exact homoclinic wave and soliton solutions for the 2D Ginzburg-Landau equation," Physics Letters A, vol. 372, no. 17, pp. 3010-3014, 2008.

[20] G. Mu and Z. Qin, "Rogue waves for the coupled schrödingerboussinesq equation and the coupled higgs equation," Journal of the Physical Society of Japan, vol. 81, no. 8, Article ID 084001, 2012.
[21] A. J. M. Jawad, M. Mirzazadeh, and A. Biswas, "Solitary wave solutions to nonlinear evolution equations in mathematical physics," Pramana-Journal of Physics, vol. 83, no. 4, pp. 457471, 2014

[22] A. J. Jawad, “Three Different Methods for New Soliton Solutions of the Generalized NLS Equation," Abstract and Applied Analysis, vol. 2017, Article ID 5137946, 8 pages, 2017.

[23] A. J. Mohamad Jawad, M. D. Petković, and A. Biswas, "Modified simple equation method for nonlinear evolution equations," Applied Mathematics and Computation, vol. 217, no. 2, pp. 869$877,2010$. 


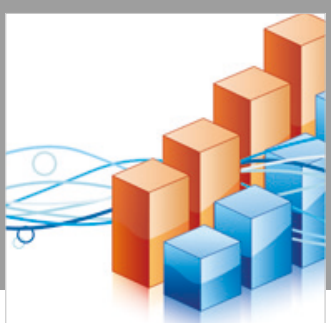

Advances in

Operations Research

\section{-n-m}
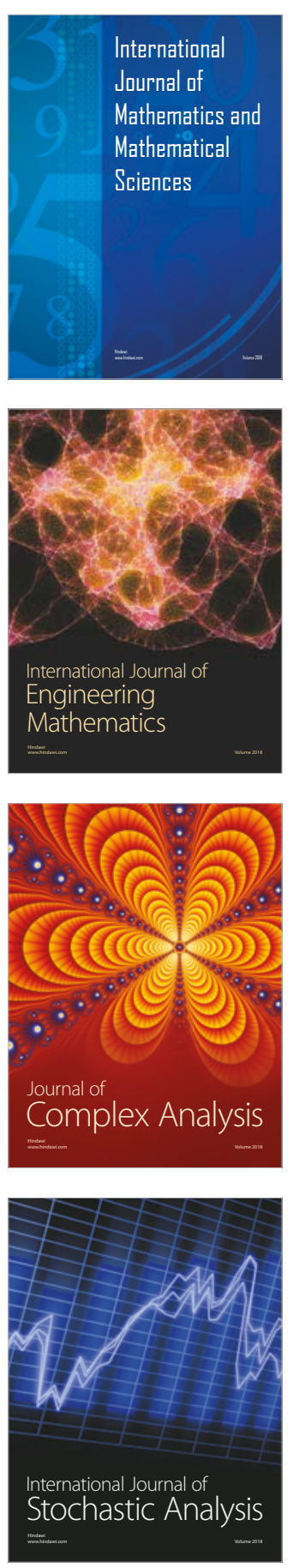
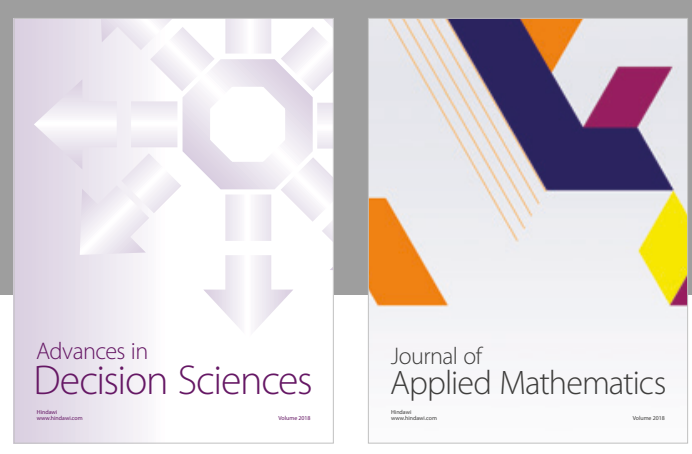

Journal of

Applied Mathematics
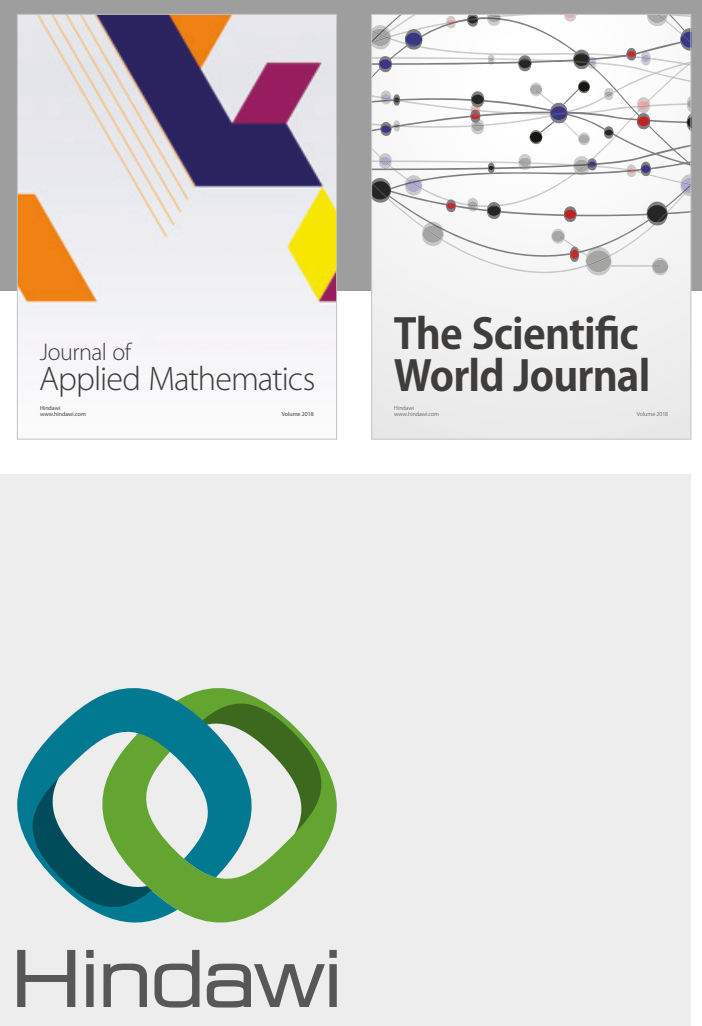

Submit your manuscripts at

www.hindawi.com

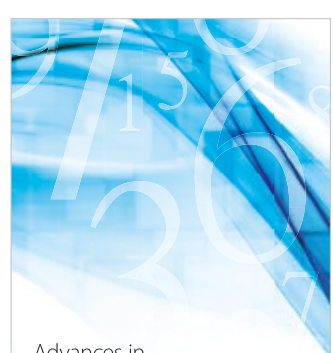

Advances in
Numerical Analysis
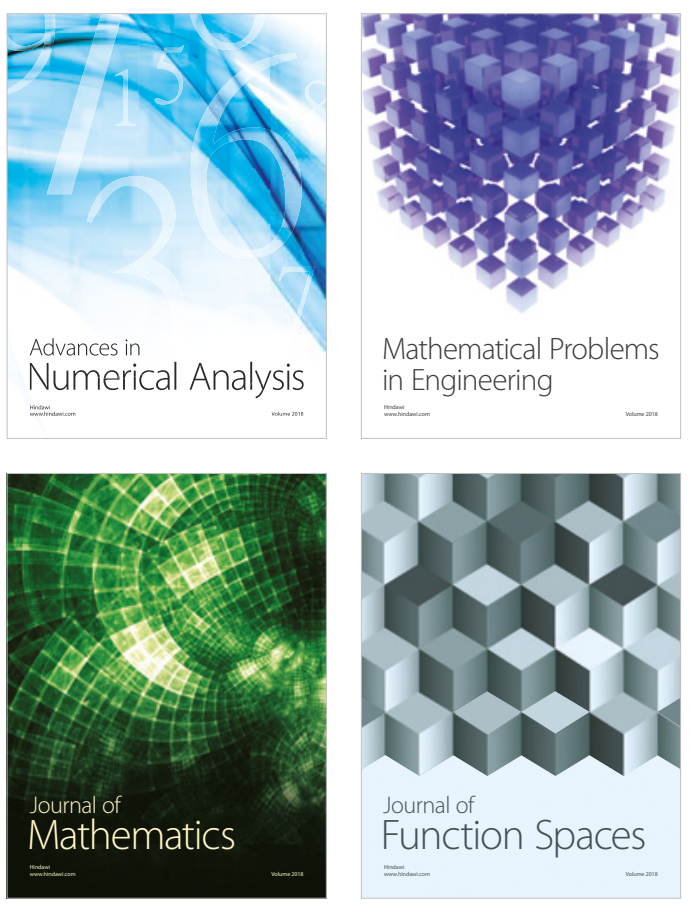

Mathematical Problems in Engineering

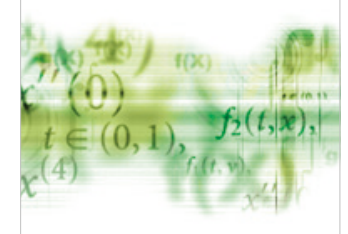

International Journal of

Differential Equations

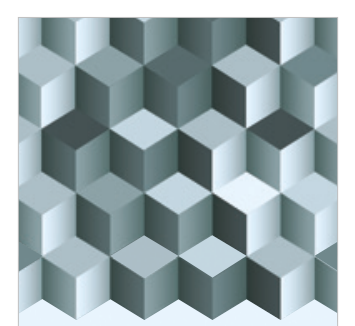

Journal of

Function Spaces

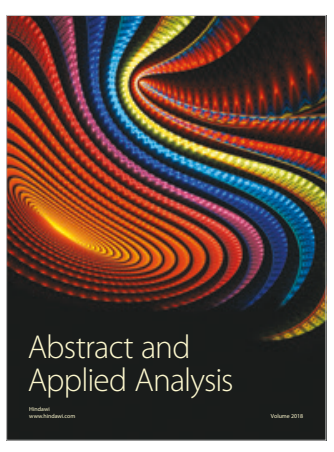

The Scientific

World Journal

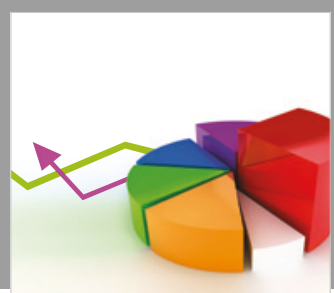

Journal of

Probability and Statistics
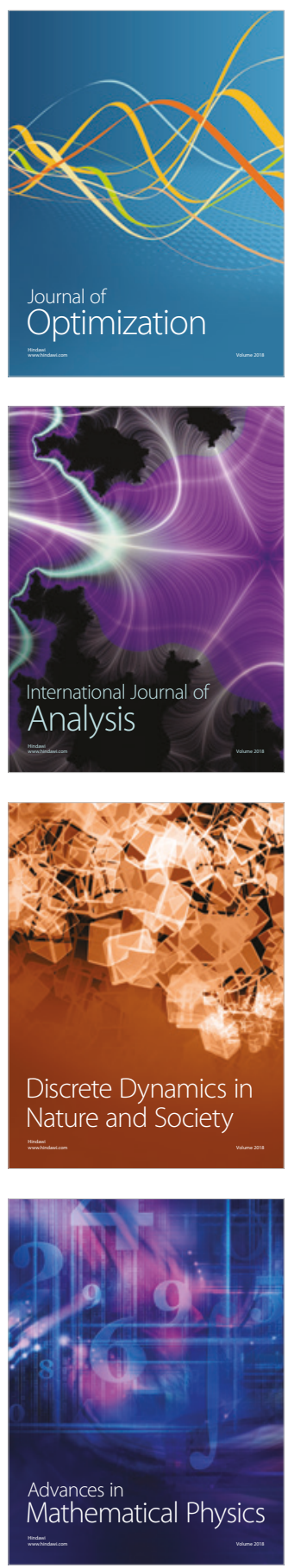